\title{
Deriving e-assessment from a competency model
}

\author{
Onjira Sitthisak Lester Gilbert Hugh C Davis \\ Learning Societies Lab \\ School of Electronics and Computer Science \\ University of Southampton, United Kingdom
}

$\{$ os05r,lg3,hcd\}@ecs.soton.ac.uk

\begin{abstract}
Self-assessment is a crucial component of learning. Creating effective questions is time-consuming, however, because it may require considerable resources and the skill of critical thinking. Questions need careful construction to accurately represent the intended learning outcome and the subject matter involved. There are very few systems currently available which generate questions automatically, and these are confined to specific domains. This paper presents a system for automatically generating questions from a competency framework, based on question templates, criteria for effective questions, and the instructional content and ability matrix. This makes it possible to guide learners in developing questions for themselves, and to provide authoring templates which speed the creation of new questions for selfassessment.
\end{abstract}

\section{Introduction}

E-assessment involves the use of a computer to support assessment, such as the use of web-based assessment tools. The importance of e-assessment for higher education and research community is well recognised [1]. Recently, the main goal of assessment has shifted away from content-based evaluation to intended learning outcome-based evaluation. This change is associated with changes in the way of questioning. The concept of competency is also increasingly important since it conceptualizes intended learning outcomes within the process of acquiring and updating knowledge throughout a learner's life [2]. This study is designed to investigate the use of a competency model to support e-assessment for lifelong learning.

In this paper, we present a system for automatically generating questions from a competency framework, named COMpetence-Based learner knowledge for personalized Assessment (COMBA). A mechanism of using question templates for generating questions is proposed. We consider an implementation of COMBA, an experiment to test its outputs, and the results. Finally, we present some discussion and conclusions.

\section{Problem statement}

Self-assessment is a crucial component of learning. Learners can learn by asking themselves questions and attempting to answer them. However, creating effective questions is time-consuming because it may require considerable resources and the skill of critical thinking [3]. Questions need careful construction to accurately represent the intended learning outcome and the subject matter involved. There are very few systems currently available which generate questions automatically, and these are confined to specific domains.

\section{System Overview}

We propose the competency model named COMpetence-Based learner knowledge for personalized Assessment (COMBA) [4]. This model focuses on the representation of competency as a rich data structure. COMBA aims to provide a system which accommodates complicated competencies, links competencies adequately, and supports tracking of the knowledge state of the learner. The system focuses on the identification and integration of appropriate subject matter content (represented by a hierarchy of competencies) and cognitive ability (represented by a capability taxonomy). This makes identification of the assessment that would demonstrate successful teaching and learning straightforward.

The system can automatically generate a list of all the questions that are possible at various levels, from a given learning outcome, the COMBA model, question templates, criteria for effective questions $[3,5]$, and the instructional content and ability matrix [6-8]. This makes it possible to guide learners in developing 
questions for themselves, and to provide authoring templates which speed the creation of new questions for self-assessment.

\section{Implementation}

COMBA consists of a number of modules (illustrated in Figure 1): competence navigator, subject matter navigator, capability navigator, question assembler, question to QTI schema converter and sequencing manipulator. The competence navigator is responsible for retrieving the requested competence based on the domain request from the user, and passing the competence to the subject matter and capability navigator modules. The relevant subject matter and capability data received from those modules, together with the authoring question template files, are assembled to generate questions derived from the matrix of competencies crossed with cognitive abilities. Then, the questions are formatted according to the IMS Question and Test Interoperability specification (QTI) ${ }^{1}$, which enables the sharing of the questions and tests. In order to develop a test, the generated questions are linked together for storing in a test bank. For the delivery of the assessment, the system deploys an assessment delivery service (ASDEL) ${ }^{2}$ to allow a learner to view a question and answer it. In the next stage of the research, the system will be extended to marking and feedback.

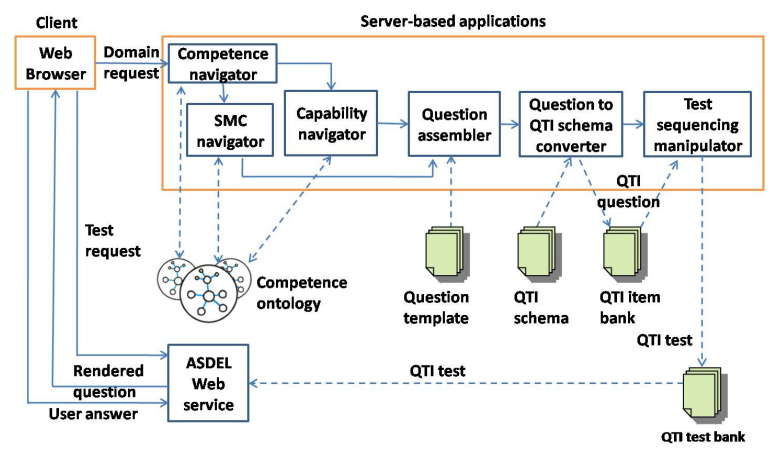

Figure 1. Architecture for the COMBA system

\section{Mechanism of generating questions}

In any unit of teaching and learning, there are usually a number of competencies intended to be achieved by learners. These competencies and their linkages may be assembled into trees. It is assumed that proficiency in all children of a defined competency

\footnotetext{
${ }^{1}$ http://www.imsglobal.org/question/qtiv2p1pd2/imsqti _oviewv2p1pd2.html

${ }^{\overline{2}}$ http://www.asdel.ecs.soton.ac.uk/
}

is a necessary precondition for achieving proficiency in the parent. While a tree defines a structure which may be traversed top-down or bottom-up, it does not imply sequencing. For example, a competency tree may illustrate the 'roll up' of the assessment for each competency throughout the tree without implying sequencing of the assessment of same level competencies. The competency tree might be used to drill down into component competencies for the target competency, helping to define what to test and how to test it.

Table 1. Question templates.

\begin{tabular}{|l|l|l|}
\hline No. & \multicolumn{1}{|c|}{ Question Templates } & $\begin{array}{c}\text { Type of } \\
\text { Question }\end{array}$ \\
\hline a & {$[$ Capability]+[Subject $]$} & Generic \\
\hline b & {$[$ Capability $]+[$ Related Subject $]$} & Generic \\
\hline c & {$[$ Capability $]+[$ Subject $]+[$ Situation $]$} & Specific \\
\hline d & $\begin{array}{l}{[\text { Capability }]+[\text { Related Subject }]+} \\
{[\text { Situation }]}\end{array}$ & Specific \\
\hline
\end{tabular}

Table 2. Example generated questions.

\begin{tabular}{|l|l|c|}
\hline $\begin{array}{l}\text { Learning } \\
\text { outcome }\end{array}$ & \multicolumn{1}{|c|}{ The questions } & $\begin{array}{c}\text { Template } \\
\text { No. }\end{array}$ \\
\hline \multirow{2}{*}{$\begin{array}{l}\text { Students } \\
\text { understand } \\
\text { the concept } \\
\text { of a } \\
\text { confidence } \\
\text { interval, } \\
\text { and can } \\
\text { calculate } \\
\text { it. }\end{array}$} & $\begin{array}{l}\text { Define the meaning of the } \\
\text { confidence interval. }\end{array}$ & $\begin{array}{l}\text { Explain the importance of the } \\
\text { critical z score. }\end{array}$ \\
$\begin{array}{l}\text { Calculate the confidence interval } \\
\text { (900) high school first year students } \\
\text { national survey. Among survey } \\
\text { participants, the mean grade-point } \\
\text { average (GPA) was 2.7, and the } \\
\text { population standard deviation was } \\
\text { 0.4 assuming a 95\% confidence } \\
\text { level. }\end{array}$ & \\
\cline { 2 - 4 } & $\begin{array}{l}\text { Explain the importance of the } \\
\text { standard error in this situation: } \\
\text { (same as above) }\end{array}$ & \\
\hline
\end{tabular}

An assessment for a competency often actually tests component competencies. For example, a statistics course may test knowledge of the confidence interval by testing the learners' ability to calculate, explain, and define the confidence interval in a variety of situations. A generic assessment item can be directly formulated from a competence specification by using the parameters of that competence: capability, subject matter content, and other elements such as the situation or context. This is illustrated as authoring question templates in Table 1. For example, the assessment corresponding to the learning outcome, "students 
understand the concept of a confidence interval" might be something like "Calculate the confidence interval for the following situation", "Explain the importance of the confidence interval in the following situation", or "Define standard error".

In the COMBA system, when learners submit their domain of interest to the system, the competence navigator module navigates the competency using the competency ontological database based on the request, where relevant subject matter and capability nodes are retrieved. In this stage, both breadth-first and depthfirst strategies could be implemented. The retrieved subject matter and capability nodes and the templates are assembled into questions. The process of traversing competencies, retrieving the relevant nodes and converting to questions are recursive. Examples of generated questions are shown in Table 2.

\section{Experiment and Results}

The experiment was designed to explore how well the generated questions rated using the criteria of clarity, usefulness, challenge, and match with the learning outcomes. The system generated 42 questions within the confidence interval topic. These questions were filtered to 25 questions based on two domain experts' selection of the questions which would most appropriately address the experimental questions. The 25 questions were classified according to their type, whether they were 'generic' questions or 'specific' questions (see Table 1).

Table 3. Mean ratings for question types.

\begin{tabular}{|l|c|c|c|}
\hline \multirow{2}{*}{ Dependent Variable } & Question type & Mean & Std. Error \\
\hline \multirow{2}{*}{ Clear } & Specific Q & 1.98 & .071 \\
\cline { 2 - 4 } & Generic Q & 1.63 & .087 \\
\hline \multirow{2}{*}{ Useful } & Specific Q & 1.63 & .071 \\
\cline { 2 - 4 } & Generic Q & 1.76 & .086 \\
\hline \multirow{2}{*}{$\begin{array}{l}\text { Match to learning } \\
\text { outcomes }\end{array}$} & Specific Q & 1.88 & .070 \\
\cline { 2 - 4 } Challenging & Generic Q & 1.78 & .086 \\
\cline { 2 - 4 } & Specific Q & 1.35 & .057 \\
\cline { 2 - 4 } & Generic Q & 1.50 & .070 \\
\hline
\end{tabular}

The study gathered data from 27 students taking the INFO1013 IT modeling course at the University of Southampton. Responses were coded as 1 (Yes), 2 (No opinion), 3 (No). Table 3 provides the means for the ratings according to question type. We found significant differences between question types in mean ratings of 'Clear' and 'Challenging', but no significant differences between question types in mean ratings of 'Useful' and 'Match to learning outcomes'. The students rated the clarity of generic questions significantly higher than that of specific questions, while rating the challenge of the specific questions significantly higher than that of the generic questions. In addition, the students rated the specific and the generic questions as not significantly different with regard to ratings of 'Useful' and 'Match to learning outcome'.

\section{Discussions and Conclusion}

This paper describes a method of automatically generating questions from a proposed competency framework to support self assessment. The results indicate that the generated questions were of acceptable value to the students. Six out of 8 of the 95\% confidence intervals excluded and were below a score of 2, indicating a tendency to rate "Yes" rather than "No opinion" or worse. Those students found the specific questions more useful and the generic questions more challenging is not an unexpected finding, and neither is the finding that both types of question did not differ significantly on the two other criteria, of their clarity and whether they matched the intended learning outcomes. The authoring question template used as the starting point in formulating the format of questions exhibited a rather low efficiency of $59.52 \%$ (the number of the generated questions, 42 , in relation to the number of selected questions, 25). It may be possible to use some natural language processing for developing the format of questions, and this point will be examined in future work.

\section{References}

[1] Matthews, B.,"Semantic Web Technologies", in JISC Technology and Standard Watch. 2005.

[2] Koper, R. and M. Specht, "TenCompetence: Lifelong Competence Development and Learning", in Competencies in Organizational E-Learning: Concepts and Tools, M.-A. Sicilia, Editor. 2007, Idea Group.

[3] McComas, W.F. and L. Abraham. "Asking more effective questions". 2005

[4] Sitthisak, O., L. Gilbert, H.C. Davis, and M. Gobbi. "Adapting health care competencies to a formal competency model". in the ICALT. 2007. Niigata, Japan: IEEE Computer Society Press.

[5] Silyn-Roberts, H., "Writing for Science". 1996, Auckland, New Zealand: Addison Wesley Longman New Zealand Limited.

[6] Kemp, J.E., G.R. Morrison, and S.M. Ross, "Designing Effective Instruction". Second Edition ed. Vol. Prentice Hall. 1998, Ohio.

[7] Merrill, M.D. "Instructional Transaction Theory (ITT): Instructional Design Based on Knowledge Objects". 1999.

[8] Krathwohl, D.R. and L. Anderson, "A revision of bloom's taxonomy: An overview". Theory into Practice, 2002. 41(4): p. 212-218. 